\title{
Spinal fusion surgery no stroke risk
}

Published online: 22 June 2012

(C) Springer Healthcare 2012

MedWire News: Spinal fusion does not significantly increase the risk for stroke in the 3 years following surgery, research from Taiwan suggests.

Indeed, initial analysis showed a trend toward a reduced risk for any stroke (hazard ratio $[\mathrm{HR}]=0.87)$, hemorrhagic stroke $(\mathrm{HR}=0.72)$, and ischemic stroke $(\mathrm{HR}=0.89)$, although no significant difference was found after adjusting for demographic data, comorbidity, and medication use.

In all, 2249 Taiwanese patients who underwent spinal surgery between 2000 and 2005 were each matched by age, gender, and comorbidity with 2203 controls, and followed up for a total of 12,967 person-years, explain Yun-Chun Chen (National Yang-Ming University Hospital, I-Lan) and co-workers.

The incidence of any stroke in the surgery and control groups was 9.95 and 11.5 events per 1000 patient-years, respectively. The corresponding rates for hemorrhagic (1.21 and 1.69) and ischemic (8.86 and 9.93) stroke were also similar between the groups.

The researchers hypothesize that the trend toward a reduced risk for stroke following spinal fusion may be accounted for by associated improvements in instability, ambulatory function, and walking. Physical activity is known to reduce the risk for stroke in a dose-dependent manner.

"It is reasonable to infer that spinal fusion surgery helps patients decrease pain and increase physical activity," Chen et al suggest in The Spine Journal.

They note that spinal surgery fusion is also often anticipated to modify other stroke risk factors, such as quitting smoking, improving diabetes controls, and treatment of arrhythmia, which may also help explain the trend toward reduction after surgery.

\section{By Lynda Williams}

\section{Reference}

Spine J 2012; Advance online publication 